\title{
CORPS ET ANNEAUX DE ROLLE ${ }^{1}$
}

\author{
FRANÇOISE DELON
}

\begin{abstract}
Brown, Craven and Pelling have proved that if the polynomials over an ordered field $K$ satisfy Rolle's theorem, they satisfy it for any ordering on $K$. We say that such a field is a Rolle field. We prove that this is a first order property in the language of rings, and that the theory of Rolle fields is decidable. Then we give a common generalisation of these fields and the real closed rings defined by Cherlin and Dickmann: The polynomials over an ordered ring $A$ satisfy Rolle's theorem iff $A$ is the valuation ring of a henselian valued field with Rolle residue field and $m$-divisible value group for all odd $m$.
\end{abstract}

I. Corps de Rolle. Pour plus de détails, le lecteur se réfèrera à [D]; d'autres propriétés logiques des corps de Rolle sont aussi étudiées dans [L].

DEFInition. Un corps ordonné $K$ a la propriété de Rolle si et seulement si, pour tout polynôme $P \in K[X]$ et pour tous $a<b$ dans $K$ vérifiant $P(a)=P(b)=0$, il existe $c$ dans $K, a<c<b$, tel que, si $P^{\prime}$ est la dérivée formelle de $P$, on ait $P^{\prime}(c)=0$.

ThEORÈmE 1 [BCP]. Le corps $K$ a la propriété de Rolle si et seulement s'il admet une valuation $v$ henselienne pour laquelle le corps de restes $K / v$ est réel clos, et le groupe $v K$ est $m$-divisible pour tout entier $m$ impair.

Ce critère est indépendant de l'ordre considéré, donc $K$ a la propriété de Rolle pour tous les ordres qu'il peut porter; cela nous amène à définir:

Definition. Un corps $K$ est un corps de Rolle si et seulement s'il est ordonnable et qu'il a la propriété de Rolle pour (un ou) tous les ordres qu'il porte.

ThEORÈmE 2. "Être un corps de Rolle" est une propriété du premier ordre dans le langage $\{0,1,+, \cdot\}$.

Demonstration. Soit $K$ un corps de Rolle, $V(K)$ l'ensemble des valuations sur $K$ ayant les propriétés du Théorème 1. Une valuation henselienne sur $K$ est nécessairement convexe (se ramener au cas d'un corps réel clos et utiliser [KW]). Cela oblige toutes les valuations de $V(K)$ à être comparables (par définition, " $v$ est plus fine que $w(v \geqslant w)$ " ssi les anneaux de valuation vérifient $A_{v} \subset A_{w}$, voir [R, $\mathrm{p}$. 54]) et la proposition suivante montre que $V(K)$ admet un élément minimal.

Received by the editors August 24, 1984 and, in revised form, January 21, 1985.

1980 Mathematics Subject Classification. Primary 03C60; Secondary 10M10, 10M15, 10N05.

Key words and phrases. Completeness, decidability, real closed ring, Rolle's theorem.

${ }^{1} \mathrm{Je}$ remercie Mme M. Giraudet et, à titre posthume, M. S. Wolfenstein, décédé le 29 septembre 1985 , organisateurs du Premier colloque international sur les structures algébriques ordonnées, (Luminy-Marseille $11 / 16$ juin 1984), où j'ai exposé les résultats contenus dans cet article. 
Proposition 1. La borne inférieure de $V(K)$ est dans $V(K)$ et est définissable au premier ordre dans la seule structure de corps de $K$.

Demonstration. Soit $v_{0}$ cette borne inférieure et $v \in V(K)$.

(1) Montrons d'abord $v_{0} \in V(K)$. On peut supposer $v_{0}<v$, sinon c'est fini. La valuation $v_{0}$ est henselienne parce que plus grossière qu'une valuation henselienne, et $v_{0} K$ est $m$-divisible pour $m$ impair parce que c'est un quotient de $v K$ qui a cette propriété; $v$ définit sur $K / v_{0}$ une valuation quotient $v / v_{0}$ (cf. [R, p. 60]) dont on voit immédiatement qu'elle est henselienne et de corps résiduel réel clos; si l'on prouve que son groupe est divisible, on en déduira que $K / v_{0}$ est est réel clos, d'où $v_{0} \in V(K)$. Soit donc $\bar{a}=a / v_{0} \in K / v_{0}, \bar{a} \neq 0$, c'est-à-dire $v_{0}(a)=0$, d'où $v_{1}(a)$ $=0$ pour $v_{1}$ assez grossière dans $V(K)$; en particulier on peut choisir $v_{1}<v$; dans ce cas $v / v_{1}$ est définie sur $K / v_{1}$ qui est réel clos et donc $\left(v / v_{1}\right)\left(K / v_{1}\right)$ est divisible; alors, quelque soit $n \in \mathbf{N}^{*}, K$ contient $b$ vérifiant

$$
n \cdot\left(v / v_{1}\right)\left(b / v_{1}\right)=\left(v / v_{1}\right)\left(a / v_{1}\right)
$$

égalité qui peut être identifiée à la suivante $n \cdot v(b)=v(a)$, d'où $n \cdot\left(v / v_{0}\right)\left(b / v_{0}\right)$ $=\left(v / v_{0}\right)\left(a / v_{0}\right)$.

(2) Remarquons que, du fait de la minimalité de $v_{0}, v / v_{0}$ est à valeurs dans le plus gros sous-groupe convexe divisible de $v K$.

(3) Venons-en à la définissabilité de $v_{0}$. On voit facilement que, pour $t \in K, v(t)$ n'est pas divisible par 2 dans $v K$ si et seulement si

$$
K \vDash \forall z\left(t \neq z^{2}\right) \wedge\left(t \neq-z^{2}\right) .
$$

Définissons (cf. [A])

$$
A(t)=\left\{x ; \exists y\left(1+t x^{2}=y^{2}\right)\right\} .
$$

Pour $v(t)$ non divisible par 2, on a

$$
A(t)=\{x ; v(x)>-v(t) / 2\} ;
$$

en conséquence, $A(t)$ est stable par multiplication si et seulement si $v(t) \leqslant 0(\Leftarrow$ est trivial; pour $\Rightarrow$ remarquer que $v K$ est dense, parce que $m$-divisible pour un entier $m \geqslant 2$, donc si $v(t)>0$ il existe $x \in K$ vérifiant $-v(t) / 2<v(x)<-v(t) / 4$, d'où $x \in A(t)$ et $\left.x^{2} \notin A(t)\right)$. Considérons maintenant l'anneau $B$ défini par

$$
\begin{aligned}
B=\{x ; & \forall t\left[\forall z\left(t \neq z^{2}\right) \wedge\left(t \neq-z^{2}\right)\right] \\
\wedge & {\left.[A(t) \text { stable par multiplication }] \rightarrow\left[x \in A\left(t^{-1}\right)\right]\right\} ; }
\end{aligned}
$$

$x$ est dans $B$ lorsque, pour tout (ou pour un) $v \in V(K)$, pour tout $t \in K$ tel que $v(t)$ soit négatif et non divisible par 2, on a $v(x)>v(t) / 2$, ou encore, si $v(x)$ est positif ou nul, modulo le sous-groupe convexe divisible maximal de $v K$, c'est-à-dire d'après le (2), exactement si $v_{0}(x) \geqslant 0$.

Le Théorème 2 se prouve alors ainsi: $K$ est un corps de Rolle si et seulement si $B$ définit dans $K$ un anneau de valuation et si, pour cette valuation $v, K$ est henselien, $K / v$ réel clos et $v K m$-divisible pour tout entier $m$ impair.

ThÉorème 3. La théorie des corps de Rolle est décidable. 
Demonstration. On utilise le principe d'Ax-Kochen-Eršov sous une forme énoncée par $V$. Weispfenning [W]: le théorème découle de la décidabilité de la théorie des corps réels clos et de la théorie des groupes abéliens ordonnés $m$-divisibles pour $m$ impair. Pour prouver cette dernière, utiliser [S1, S2, S3].

De la même façon, le théorème suivant se déduit du résultat analogue pour les groupes abéliens ordonnés.

THÉORÈE 4. Il y a $2^{\aleph_{0}}$ théories complètes de corps de Rolle, dont $\boldsymbol{\aleph}_{0}$ décidables.

Pour préciser la description algébrique des corps de Rolle, citons les résultats suivants.

Proposition 2 [BCP]. Un corps ordonné $K$ a la propriété de Rolle si et seulement si la valuation sur $K$ qui est convexe et de corps de restes archimédien, est dans $V(K)$.

Proposition 3. Si K est un corps de Rolle et $v$ une valuation sur $K$, les propositions suivantes sont équivalentes:

(1) $v$ est convexe pour un ordre de $K$,

(2) $v$ est convexe pour tout ordre de $K$,

(3) $v$ est henselienne,

(4) $K / v$ est un corps de Rolle,

(5) $K / v$ est ordonnable,

(6) $K / v$ n'est pas algébriquement clos si $\operatorname{car}(K / v) \neq 2, K / v$ n'est pas séparablement clos sinon.

Démonstration. Lorsque $K$ est réel clos, on a $1 \leftrightarrow 3 \leftrightarrow 5 \leftrightarrow K / v$ non algébriquement clos; utiliser ces équivalences, la Proposition 2, le fait que dans un corps henselien le corps de restes se relève, s'il est de caractéristique nulle, et le fait qu'un corps henselien pour deux valuations indépendantes est séparablement clos.

II. Anneaux de Rolle. "Anneau” signifiera toujours "anneau commutatif, intègre et unitaire".

Définition. Un anneau ordonné $A$ a la propriété de Rolle lorsque, pour tout $P \in A[X]$ et $a<b$ des racines de $P$ dans $A, P^{\prime}$ admet un zéro $c$ vérifiant $a<c<b$.

Il y a une seule façon de prolonger l'ordre de $A$ à son corps de quotients $Q(A)$ et on a le fait suivant:

Lemme 1. Si $(A,<)$ a la propriété de Rolle, $(Q(A),<)$ l'a aussi.

Démonstration sans problème.

Remarque. Pour appliquer la propriété de Rolle dans $A$, peu importe que $P$ soit à coefficients dans $A$ ou $Q(A)$; seul le fait que les racines $a$ et $b$ soient dans $A$ est important.

Lemme 2. Le groupe additif de A est divisible; en particulier A contient $\mathbf{Q}$. 
Démonstration. Pour $a \in A^{*}$, la dérivée de $X^{n}-a X^{n-1}$ doit avoir une racine strictement comprise entre 0 et $a$, ce ne peut être que $a-a / n$, donc $a / n$ est dans A.

Corollaire 1. Pour $P \in A[X]$ et $a<b$ dans $A$, on $a \int_{a}^{b} P(x) d x \in A$.

Dans la suite, $v$ est l'unique valuation sur $Q(A)$ convexe et telle que $Q(A) / v$ soit archimédien, donc se plonge dans $\mathbf{R}$.

Proposition 4. Soit $P \in\left(A \cap A_{v}\right)[X]$ tel que $P / v$ admette une racine réelle $d$ 'ordre impair; alors cette racine se relève dans $A \cap A_{v}$ en une racine de $P$.

Demonstration. La même que dans $[\mathbf{B C P}]$ pour prouver 2-1 $(\mathrm{a} \Rightarrow \mathrm{c})$, grâce au fait que $A$ contient $\mathbf{Q}$ et à notre Corollaire 1 .

Lemme 3. Si $a$ et $b \in A$ sont tels qu'il existe $c \in A$ vérifiant $v(a) \geqslant v(b)=-v(c)$, alors $a b^{-1} \in A$; en particulier $v(a) \geqslant v(b)=0$ implique $a b^{-1} \in A$.

Demonstration. On applique la Proposition 4 au polynôme $(b X-a) c$.

Proposition 5. $A_{v} \subset A$.

Demonstration. Soit $c \in Q(A), c>0$. Supposons d'abord $c<1$, donc $v(c) \geqslant 0$;

(1) $c / v \neq 1 / 2$. On cherche dans $Q(A)$ un polynôme de degré $3, P(X)=X^{3}+$ $x X^{2}+y X$ s'annulant en 0 et 1 et dont la dérivée s'annule en $c$; cela conduit au système

$$
1+x+y=0, \quad 3 c^{2}+2 x c+y=0
$$

de déterminant $1-2 c \neq 0$ car $c / v \neq 1 / 2$; d'après la propriété de Rolle, soit $c$ soit l'autre racine $d$ de $P^{\prime}$ est dans $A$; or si $d \in A, c$ va être aussi dans $A$ grâce au Lemme 3; en effet

$$
c=-2 x / 3-d=(2-3 d)(3-6 d)^{-1}
$$

où $d$ vérifie l'égalité symétrique

$$
d=(2-3 c)(3-6 c)^{-1}
$$

et est donc de valuation $\geqslant 0$ avec $d / v \neq 1 / 2$.

(2) Si $c / v=1 / 2$, on change $P$, par exemple on cherche un polynôme s'annulant en 0 et 2 , et on fait le même raisonnement.

Si on suppose maintenant $c \geqslant 1$ et $c$ dans $A_{v}$, puisque $A_{v}$ est la clôture convexe de $\mathbf{Q}$ dans $Q(A)$, il existe un entier $n>c$; alors $c n^{-1}$ est dans $A$ par le raisonnement précédent, donc aussi $c$.

Corollaire 2. $(A,<)$ a la propriété de Rolle si et seulement si $(Q(A),<) l$ 'a et si $A$ contient $A_{v}$.

Démonstration. Il nous reste à vérifier l'implication "si". Parce que $A$ contient $A_{v}$, il est lui-même convexe dans $Q(A)$; il a donc la propriété de Rolle dès que $Q(A)$ l'a. 
En conséquence un anneau a la propriété de Rolle simultanément pour tous les ordres qu'il peut porter.

DEFINITION. On appelle anneau de Rolle un anneau ordonnable ayant la propriété de Rolle pour (un ou) tous ses ordres.

Nous donnons une caractérisation des anneau de Rolle parallèle au Théorème 1 et indiquant aussitôt quels sont les invariants logiques d'un tel anneau.

ThÉrème 5. A est un anneaux de Rolle si et seulement s'il est l'anneau d'une valuation $w$ henselienne avec $w(Q(A))$ m-divisible pour tout $m$ impair et $Q(A) / w$ corps de Rolle.

Demonstration. (1) Preuve de "seulement si". Si $A$ est un anneau de Rolle, $Q(A)$ est un corps de Rolle et $A$ contient $A_{v}$, c'est donc l'anneau d'une valuation $w$ plus grossière que $v$ et, par conséquent, henselienne. Par la Proposition $3, Q(A) / w$ est un corps de Rolle. Enfin $w(Q(A))$ est $m$-divisible pour tout $m$ impair, parce que c'est un quotient de $v(Q(A))$ qui a cette propriété.

(2) Preuve de "si". $Q(A) / w$ étant un corps de Rolle, il est henselien pour une valuation $\bar{v}$ dont le groupe est $m$-divisible pour tout $m$ impair et le corps de restes réel clos; sur $Q(A)$ la valuation composée (cf. [R, p. 62]) de $w$ et $\bar{v}$ a elle-même les propriétés du Théorème $1 ; Q(A)$ est donc un corps de Rolle, et $A$, qui est convexe dans $Q(A)$ pour n'importe quel ordre, est un anneau de Rolle.

ThEORÈmE 6. "Être un anneau de Rolle" est une propriété du premier ordre dans le langage des anneaux. La théorie des anneaux de Rolle est décidable.

Demonstration. Conséquence de ce qui précède et du principe d'Ax-KochenEršov.

\section{BiBLIOGRAPHIE}

[A] J. Ax, On the undecidability of power series fields, Proc. Amer. Math. Soc. 16 (1965), 846.

[BCP] R. Brown, T. C. Craven et M. J. Pelling, Ordered fields satisfying Rolle's theorem, Illinois J. Math. (à paraître).

[CDi] G. Cherlin et M. A. Dickmann, Real closed rings, Ann. Pure Appl. Logic 25 (1983), 213-231.

[D] F. Delon, Rolle fields and rings, Communication au Premier Colloque International sur les Structures Algébriques Ordonnées, Luminy-Marseille, Juin 1984.

[KW] M. Knebusch et M. J. Wright, Bewertungen mit reeller Henselisierung, J. Reine Angew. Math. 286-7 (1976), 314-321.

[L] B. Laslandes, Théorie des modèles des corps n-ordonnés, Thèse de 3ème cycle, Univ. Paris 7, Juin 1984.

[R] P. Ribenboim, Théorie des valuations, Presses Univ. Montréal, Montréal, 1964.

[S1] P. Schmitt, Model theory of ordered abelian groups, Habilitationsschrift, Heidelberg, 1982.

[S2] __ Elementary properties of ordered abelian groups (à paraître).

[S3] _ Model- and substructure complete theories of ordered abelian groups, Models and Sets

(G.H. Müller and M. M. Richter, eds.), Lecture Notes in Math., vol. 1103, Springer-Verlag, Berlin, 1984. pp. $389-418$.

[W] V. Weispfenning, Elementary theory of Hensel fields, Ann. Math. Logic 10 (1976), 59-93.

Universite Paris 7, U.E.R. De Mathematique et Informatique, 2 Place Jussieu, 75251 Paris CEDEX 05, FRANCE 